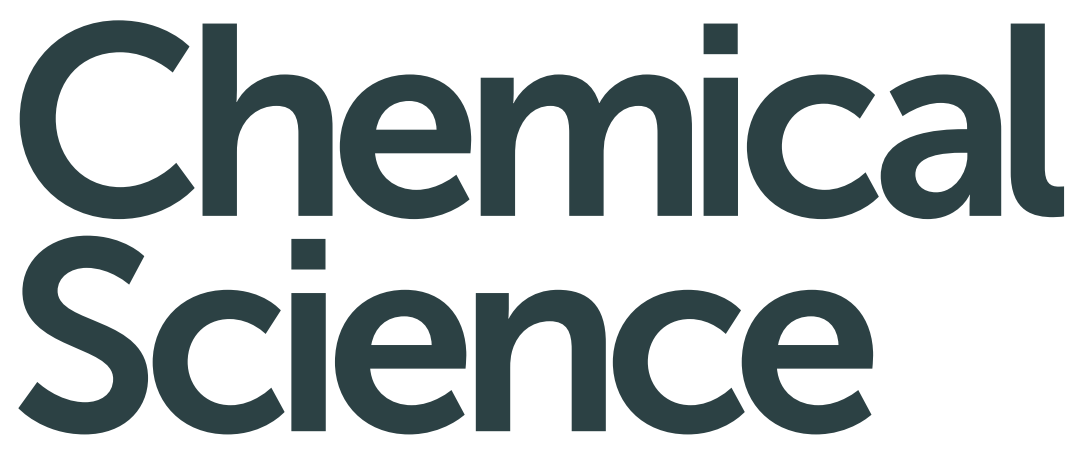

rsc.li/chemical-science
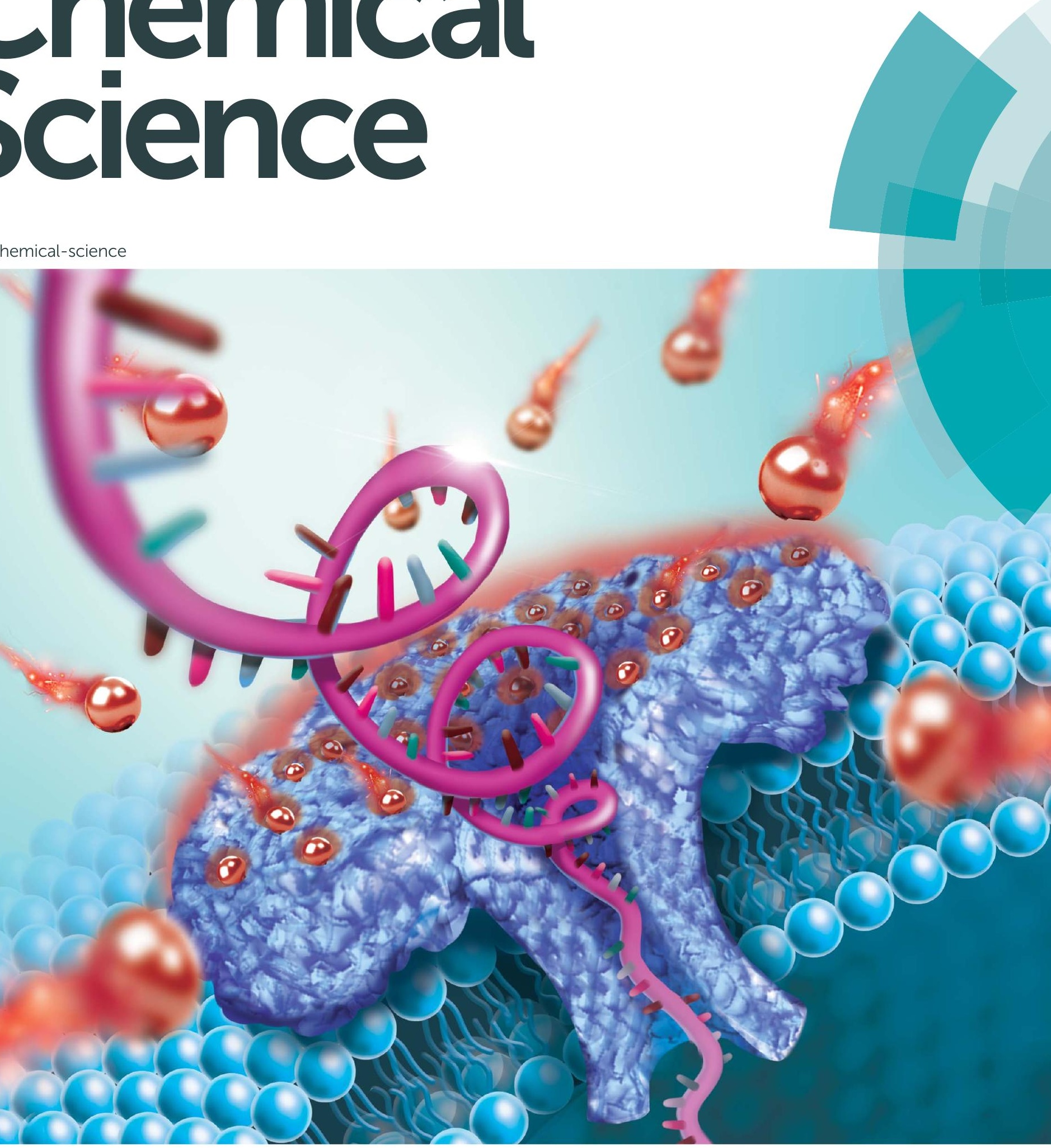

ISSN 2041-6539

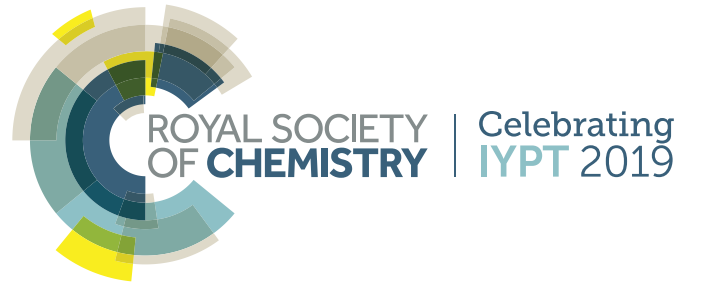


Check for updates

Cite this: Chem. Sci., 2019, 10, 354

๑ All publication charges for this article have been paid for by the Royal Society of Chemistry

Received 3rd September 2018 Accepted 12th November 2018

DOI: $10.1039 / \mathrm{c} 8 \mathrm{sc} 03927 \mathrm{e}$

rsc.li/chemical-science

\section{A lithium-ion-active aerolysin nanopore for effectively trapping long single-stranded DNA $\uparrow$}

\author{
Zheng-Li Hu, Meng-Yin Li, Shao-Chuang Liu, Yi-Lun Ying (D) and Yi-Tao Long (D)
}

Wild-type aerolysin (AeL) nanopores allow direct single nucleotide discrimination of very short oligonucleotides ( $\leq 10 \mathrm{nt}$ ) without labelling, which shows great potential for DNA sensing. To achieve real applications, one major obstacle of AeL is its poor capture ability of long single-stranded DNA (ssDNA, >10 nt). Here, we have proposed a novel and robust strategy for the electrostatic focusing of long ssDNA into a lithium-chloride ( $\mathrm{LiCl}$ )-active AeL. By using this method, for the first time we have demonstrated AeL detection of ssDNA longer than $100 \mathrm{nt}$. Due to screening more negative charges, $\mathrm{LiCl}$ improves AeL capture ability of long ssDNA (i.e. $60 \mathrm{nt}$ ) by 2.63- to 10.23-fold compared to $\mathrm{KCl}$. Further calculations and molecular dynamics simulations revealed that strong binding between $\mathrm{Li}^{+}$and the negatively charged residue neutralized the AeL, leading to a reduction in the energy barrier for ssDNA capture. These findings facilitate the future high-throughput applications of AeL in genetic and epigenetic diagnostics.

\section{Introduction}

Nanopore techniques have been developed into powerful platforms for single molecule sensing in a rapid and low-cost way. Aerolysin (AeL), a rivet-shaped protein nanopore with an inner diameter of $\sim 1.0 \mathrm{~nm}$, was initially used to investigate the structural variation of $\alpha$-helical peptides, ${ }^{\mathbf{1}}$ then applied to discriminate polymer sizes, study protein folding/unfolding, describe enzyme kinetics and monitor chemical bond making/breaking. ${ }^{2-6}$ In particular, AeL demonstrated its superior resolution in discrimination of very short oligonucleotides ( $\leq 10 \mathrm{nt}) .^{7-9}$ The high sensitivity and long reads are the two important parameters for DNA sensing and sequencing. Compared with other biological nanopores such as $\alpha$-hemolysin $(\alpha-\mathrm{HL})$ and Mycobacterium smegmatis porin A (MspA), ${ }^{\mathbf{1 0}-\mathbf{1 3}} \mathrm{AeL}$ has high sensitivity in direct discrimination of single nucleotides without chemical labelling, the incorporation of molecular motors or recognition probes., ${ }^{\mathbf{9} 14}$ Previous work showed that AeL was able to recognise subtle differences between methylated cytosine and cytosine under serum conditions. ${ }^{\mathbf{1 4}}$ Therefore, AeL shows great potential for direct DNA sensing and sequencing in a simple and cheap manner. However, all these achievements were based on very short oligonucleotides $(\leq 10$ nt). Few real applications have been achieved via AeL because it is difficult for long single-stranded DNA (ssDNA, >10 nt) to enter

Key Laboratory for Advanced Materials, School of Chemistry and Molecular Engineering, East China University of Science and Technology, Shanghai, 200237, P. R. China. E-mail: yilunying@ecust.edu.cn; Tel: +86-21-64252339

$\dagger$ Electronic supplementary information (ESI) available: Protocol for aerolysin nanopore experiment, molecular dynamics simulations, histograms of the event frequencies and durations, and supporting figures. See DOI: 10.1039/c8sc03927e into the AeL. For instance, AeL analysis of a 50 nt ssDNA shows an event frequency as low as $\sim 0.8 \mathrm{~s}^{-1}$ at $100 \mathrm{mV}$ at a concentration of $2.0 \mu \mathrm{M} .{ }^{15}$ Most recently, our study demonstrated the discrimination of A, T, C and $\mathrm{G}$ in a 14 nt oligonucleotide but showed a low AeL capture rate of the ssDNA. ${ }^{16}$ This challenge largely restricts nanopore techniques from achieving direct sensing of long stranded DNA. To improve the capture ability of AeL for long stranded DNA, several strategies have been proposed. By increasing the applied voltage to a value as high as $70 \mathrm{mV}$, a $60 \mathrm{nt}$ ssDNA showed an increased event frequency to $0.4 \mathrm{~s}^{-1} \cdot{ }^{17}$ In our previous work, by modulating the G-quadruplex DNA into an extended form using magnesium ions $\left(\mathrm{Mg}^{2+}\right)$, the subsequent capture of a $30 \mathrm{nt}$ structured DNA was observed at an event frequency of $0.6 \mathrm{~s}^{-1} \cdot{ }^{18}$ By raising the temperature from 20 to $60^{\circ} \mathrm{C}$, the event frequency of a $50 \mathrm{nt}$ ssDNA increased from 0.8 to $2.2 \mathrm{~s}^{-1} \cdot{ }^{15}$ By protonating AeL in an asymmetric $\mathrm{pH}$ environment, the translocation of a $16 \mathrm{nt}$ ssDNA was improved to $5.6 \mathrm{~s}^{-1} \cdot{ }^{19}$ However, these methods increase the difficulty of the experiment and reduce the stability of the sensing system. The high voltage destabilizes the lipid bilayer membrane, and the high temperature causes variation in the salt concentration due to the non-negligible evaporation of water and might irreversibly denature the protein nanopores. The asymmetric $\mathrm{pH}$ environment induces spontaneous current blocks for AeL at applied voltages higher than $100 \mathrm{mV} \cdot{ }^{19}$ Thus, a novel and robust approach is urgently needed to improve AeL's ability to capture long stranded DNA.

According to previous studies, ${ }^{19-24}$ various possible effects could modulate DNA translocation dynamics, such as the geometry of the nanopore, the $\mathrm{pH}$ of the solution, the salt type and the electrolyte concentration gradient. These effects mainly 
regulate the energy barrier for the entry of DNA, in particular they reduce the interaction between DNA and the entrance of the nanopores. As for aerolysin nanopores, they emerge as negatively-charged protein nanopores $(-52 \mathrm{e})$ with numerous aspartic (Asp) and glutamic (Glu) residues around the cis entrances, such as Asp216, Asp207, Glu307 and Glu415. ${ }^{25}$ The strong electrostatic repulsion between the negatively charged nucleotides and the amino acids at the entrance contributes to a large energy barrier for DNA entering into the AeL. Therefore, a potential solution is to reduce the negative charges at the cis entrance of the AeL. The local charge distribution of a biological nanopore could be changed using site-directed mutagenesis and chemical modification. ${ }^{26-28}$ However, these methods are always complicated and time-consuming. Previous studies have demonstrated that lithium ions $\left(\mathrm{Li}^{+}\right)$exhibit a specific preference over other alkali metal ions due to their high charge density and small size. ${ }^{23,29} \mathrm{As} \mathrm{Li}^{+}$shows a stronger binding affinity and a longer binding time with charged residues (i.e. Asp) compared with other alkali metal ions,${ }^{30}$ it could be used to screen the negative charges of a biological nanopore. Herein, we adopted lithium chloride ( $\mathrm{LiCl}$ ) to improve AeL's ability to capture long ssDNA. It has been reported for the first time that AeL detection of a $102 \mathrm{nt}$ DNA (ssDNA_102) could be achieved in LiCl. Compared with $\mathrm{KCl}$, LiCl enhanced the capture ability of AeL for ssDNA_102 by 3.89 to 9.07 fold.

\section{Results and discussion}

First, we demonstrated that the presented LiCl sensing system shows high stability in a wide voltage range (Fig. S1†). Fig. 1 illustrates a scheme of the nanopore experiment, depicting an individual ssDNA molecule captured in an AeL nanopore in $\mathrm{M}^{+} \mathrm{Cl}^{-}\left(\mathrm{M}^{+}=\mathrm{Li}^{+}\right.$or $\left.\mathrm{K}^{+}\right)$. For both electrolytes, we added the ssDNA in the cis side of the AeL, where the applied voltage is termed as grounded. Here, we adopted the event frequency as a surrogate of capture ability, which is defined as the number of blockades per second and evaluated through the inverse fitting of interevent time. We performed the assays of ssDNA_102 and obtained its real-time current traces in $\mathrm{LiCl}$ and $\mathrm{KCl}$. The duration times of ssDNA_102 decrease as the applied voltage ranges from 100 to $160 \mathrm{mV}$, indicating the successful translocations of ssDNA_102 in $\mathrm{M}^{+} \mathrm{Cl}^{-}\left(\mathrm{M}^{+}=\mathrm{Li}^{+}\right.$or $\left.\mathrm{K}^{+}\right)$(Fig. 1). Although the signals exhibit similar full blockades, the event frequencies of ssDNA_102 increase apparently when the electrolyte is changed from $\mathrm{KCl}$ to $\mathrm{LiCl}$. The event frequency of ssDNA_102 in LiCl at $100 \mathrm{mV}$ is $2.46 \pm 0.11 \mathrm{~s}^{-1}$, producing a 9.07-fold increase compared to that in $\mathrm{KCl}\left(0.27 \pm 0.04 \mathrm{~s}^{-1}\right)$ (Fig. 1 and $\mathrm{S} 2 \dagger$ ). When the applied voltage is increased to $160 \mathrm{mV}$, the event frequency of ssDNA_102 further increases from $2.26 \pm 0.23 \mathrm{~s}^{-1}$ in $\mathrm{KCl}$ to $8.79 \pm 0.54 \mathrm{~s}^{-1}$ in LiCl (Fig. 1 and $\mathrm{S} 2 \dagger)$.

To figure out the enhancement mechanism in $\mathrm{LiCl}$, we studied the behavior of polydeoxyadenines $\left(\mathrm{dA}_{n}\right)$ with different lengths in $\mathrm{M}^{+} \mathrm{Cl}^{-}\left(\mathrm{M}^{+}=\mathrm{Li}^{+}\right.$or $\left.\mathrm{K}^{+}\right)$. In LiCl, $\mathrm{dA}_{20}$ and $\mathrm{dA}_{40}$ exhibit a better linear frequency-voltage dependence, suggesting a more dominant diffusion-controlled capture process (Fig. 2ac). ${ }^{19,31,32}$ However, $\mathrm{dA}_{60}$ shows an exponential frequency-voltage
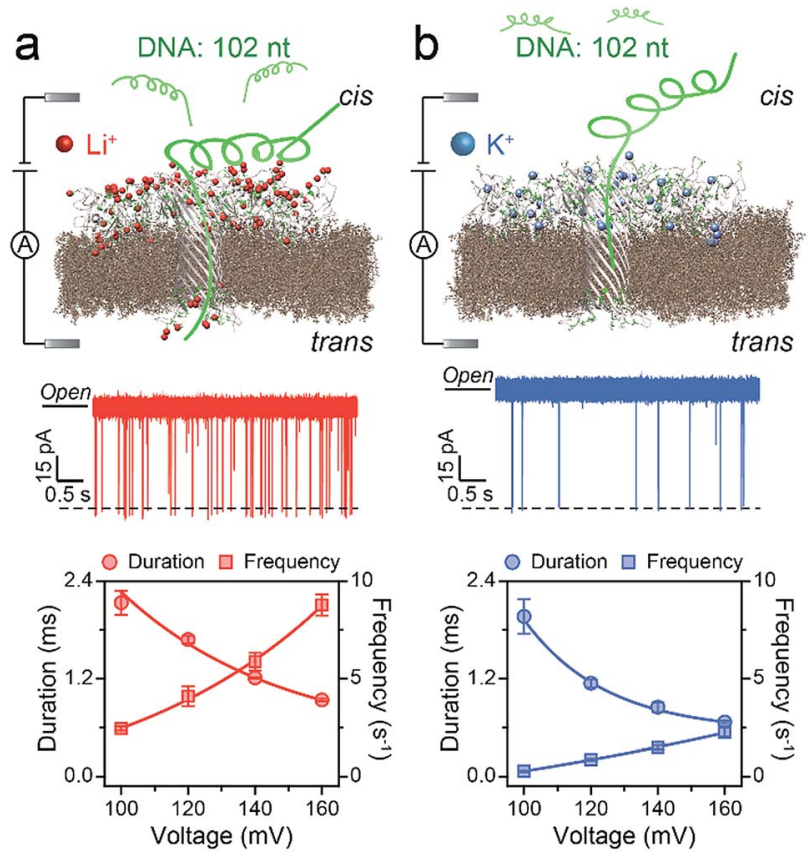

Fig. 1 Illustration of the detection of a 102 nt ssDNA (ssDNA_102) using an AeL nanopore in $\mathrm{M}^{+} \mathrm{Cl}^{-}\left(\mathrm{M}^{+}=\mathrm{Li}^{+}\right.$or $\left.\mathrm{K}^{+}\right)$. Top: the electrostatic attraction in $\mathrm{LiCl}$ and the relative electrostatic repulsion in $\mathrm{KCl}$ of a single ssDNA_102 molecule into AeL. The illustration is not to scale. Middle: raw current traces of ssDNA_102. More frequent blockade events were observed clearly in $\mathrm{LiCl}$ compared with $\mathrm{KCl}$ from the realtime current traces. Bottom: voltage dependence of SSDNA_102 translocation. Data in this work were obtained in $1 \mathrm{M} \mathrm{M}^{+} \mathrm{Cl}^{-}\left(\mathrm{M}^{+}=\mathrm{Li}^{+}\right.$ or $\mathrm{K}^{+}$), $1 \mathrm{mM}$ EDTA, $10 \mathrm{mM}$ Tris buffer, and $\mathrm{pH} 8.0$ with the presence of $2.0 \mu \mathrm{M}$ ssDNA at the cis side of the AeL nanopore.

relationship, indicating a barrier-limited capture process (Fig. 2c). These results unravel the length-dependent capture of $\mathrm{dA}_{n}$ in LiCl: diffusion is predominantly responsible for short stranded $\mathrm{dA}_{n}$ and an energy barrier is primarily accountable for long stranded $\mathrm{dA}_{n}$. As expected, similar results were obtained for $\mathrm{dA}_{n}$ in $\mathrm{KCl}$ (Fig. 2a-c). ${ }^{7}$ According to previous studies, ${ }^{33,34}$ the energy barrier for DNA capture mainly originates from DNA entropy and DNA-pore electrostatic interactions at the pore entrance. As the length of $\mathrm{dA}_{n}$ increases, entropy likely contributes to a portion of the energy barrier for the capture of long stranded $\mathrm{dA}_{n}$. Therefore, the event frequencies reduce from $8.75 \pm 0.06$ to $5.81 \pm 0.31 \mathrm{~s}^{-1}$ with $\mathrm{dA}_{n}$ elongating from 20 to $60 \mathrm{nt}$ at $160 \mathrm{mV}$ in $\mathrm{LiCl}$ due to an increased energy barrier. Interestingly, LiCl induces higher event frequencies for $\mathrm{dA}_{n}(n=$ $20,40,60)$ than $\mathrm{KCl}$ at the same applied voltage and the same DNA concentration. LiCl caused a 2.63- to 10.23-fold enhanced capture of $\mathrm{dA}_{n}(n=20,40,60)$ compared to that obtained in KCl. The possible explanation could be a lower energy barrier at the cis entrance of the AeL for $\mathrm{dA}_{n}$ capture in $\mathrm{LiCl}$ than in $\mathrm{KCl}$. $\mathrm{As} \mathrm{Li}^{+}$ screens more negative charges than $\mathrm{K}^{+}$, we deduced that the lower energy barrier in LiCl results from weaker DNA-AeL electrostatic repulsions at the cis entrance of the pore.

To demonstrate this speculation, we evaluated the electrolyte-dependent difference in energy barriers for $\mathrm{dA}_{60}$, which shows the barrier-limited capture both in LiCl and KCl. 

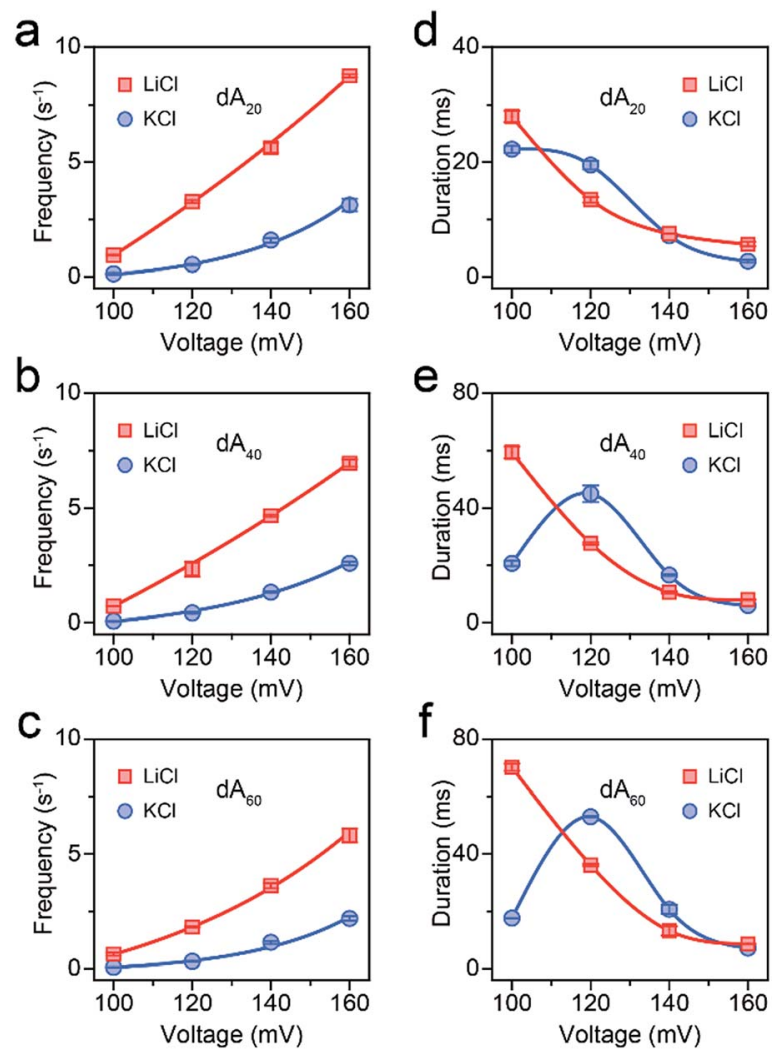

Fig. 2 AeL detection of $\mathrm{dA}_{n}$ with different lengths in $\mathrm{M}^{+} \mathrm{Cl}^{-}\left(\mathrm{M}^{+}=\mathrm{Li}^{+}\right.$ or $\mathrm{K}^{+}$). Voltage-dependent $(\mathrm{a}-\mathrm{c})$ event frequencies and $(\mathrm{d}-\mathrm{e})$ blockade durations of $\mathrm{dA}_{n}(n=20,40,60)$ in $\mathrm{LiCl}$ (red squares) and $\mathrm{KCl}$ (blue circles). The values of event frequencies and blockade durations were evaluated from transformed single-exponential functions (Fig. S3S5†).

The event frequencies of $\mathrm{dA}_{60}$ shown in Fig. 2c are well-fitted with eqn (1):

$$
f=f_{0} \mathrm{e}^{V / V_{0}}
$$

where $V$ is the applied voltage, $V_{0}$ is the applied voltage for a charged polymer to overcome Brownian motion, and $f_{0}$ is an extrapolated frequency in the absence of the applied voltage. ${ }^{33,34}$ According to the Van't Hoff-Arrhenius law, $f_{0}$ was calculated by eqn (2):

$$
f_{0}=\frac{C D A}{L} \times \mathrm{e}^{\frac{-U}{k_{\mathrm{B}} T}}
$$

where $U$ is the activation energy, $k_{\mathrm{B}}$ is the Boltzmann constant, $T$ is the temperature, $C$ is the concentration of the analyte in the bulk solution, $D$ is the diffusion coefficient of the analyte, $A$ is the sectional area of the pore and $L$ is the pore length..$^{33,34}$ The values of $f_{0}$ for $\mathrm{dA}_{60}$ are $5.29 \times 10^{-3}$ in $\mathrm{KCl}$ and $4.65 \times 10^{-1}$ in $\mathrm{LiCl}$, respectively. Assuming $D(\mathrm{LiCl}) \approx D(\mathrm{KCl})$, we obtained a reduction in the activation energy of $4.48 k_{\mathrm{B}} T$ from LiCl to KCl for $\mathrm{dA}_{60}$. Fig. $2 \mathrm{~d}-\mathrm{f}$ display hill-shaped duration-voltage curves and a threshold voltage of $120 \mathrm{mV}$ for $\mathrm{dA}_{n}(n=20,40,60)$ in KCl, indicating $\mathrm{dA}_{n}(n=20,40,60)$ could not traverse across the AeL in $\mathrm{KCl}$ at a potential lower than $120 \mathrm{mV}$. The duration time for a successful translocation is composed of the residence time of ssDNA inside the AeL and the translocation time of ssDNA through the AeL. Meanwhile for failed translocation, the duration time is only composed of the residence time of DNA inside the AeL. By increasing the applied voltage, we enhanced the electric driving force to overcome the energy barrier for the successful translocation of DNA. ${ }^{29}$ As a result, the maximum duration times observed at the threshold voltage of $120 \mathrm{mV}$ were $45.00 \pm 2.95 \mathrm{~ms}$ for $\mathrm{dA}_{40}$ and $52.98 \pm 0.26 \mathrm{~ms}$ for $\mathrm{dA}_{60}$ in KCl. Then, the duration times for $\mathrm{dA}_{n}(n=20,40,60)$ decrease for applied potentials above $120 \mathrm{mV}$ (Fig. 2a-c). In line with previous discussion, as $\mathrm{Li}^{+}$lowers the energy barrier by screening more negative charges than $\mathrm{K}^{+}$, the threshold voltage is greatly reduced for DNA capture in LiCl. Therefore, a monotonic exponential duration-voltage relation was obtained in LiCl for $\mathrm{dA}_{n}(n=20,40,60)$ over the whole voltage range. The DNA translocation speed may be modulated by various factors. Note that the duration time of $\mathrm{dA}_{n}(n=20,40,60)$ is determined by multiple non-covalent interactions between the pore and analyte. Our previous work revealed that the electrostatic interactions between AeL and ssDNA are not the only crucial factors in determining the DNA translocation speed. ${ }^{28}$ Due to the small volume of the AeL, only a small amount of $\mathrm{Li}^{+} / \mathrm{K}^{+}$can be accommodated inside the confined nanopore space. Therefore, the charge screening of ssDNA and/or AeL induced by counterions does not dominate DNA translocation speed. Synergistic coeffects including van der Waals forces, hydrogen bonding and geometrical structures will also lead to duration differences for ssDNA, and are under study in our future work.

To better understand the capture enhancement mechanism, we performed molecular dynamics (MD) simulations in $\mathrm{M}^{+} \mathrm{Cl}^{-}$ $\left(\mathrm{M}^{+}=\mathrm{Li}^{+}\right.$or $\left.\mathrm{K}^{+}\right)$. The cations are defined as being bound within a cutoff distance of $3.1 \AA$ from the heavy atoms of the pore. ${ }^{23}$ Fig. 3a illustrates the distributions of the bound $\mathrm{Li}^{+}$, viewed from the cis side of the AeL $\left(\mathrm{K}^{+}\right.$-AeL binding is shown in Fig. S6 $\dagger$ ). The binding number of $\mathrm{Li}^{+} / \mathrm{K}^{+}$was averaged over a 10 ns MD simulation, fitting into a Gaussian distribution (Fig. 3b). The average binding numbers are 194 for $\mathrm{Li}^{+}$and 87 for $\mathrm{K}^{+}$ (Fig. 3b). Although AeL exhibits an identical net charge in the two different electrolytes, the effective charges change due to the cation-AeL electrostatic interactions (Fig. 3a and S6 ${ }^{\dagger}$ ). The different $\mathrm{Li}^{+} / \mathrm{K}^{+}$-AeL binding induced variation of the electrostatic potential distributions at the cis entrance of the pore. Since the $\mathrm{Li}^{+} / \mathrm{K}^{+}$ions created a local concentration of positive ions in the pore cap, the pore vicinity is effectively polarized and the electrostatic potential increases. ${ }^{31,35}$ In KCl, AeL presents a highly negative electrostatic potential in a confined region at the cis entrance of the pore (Fig. 3c). However, a highly positive electrostatic potential occupies most areas of the pore cap in LiCl (Fig. 3c). As ssDNA is negatively charged, the high positive electrostatic potential facilitates the capture of ssDNA from the cis entrance of AeL in $\mathrm{LiCl}$, in good accordance with our previous discussion.

Furthermore, we employed $\mathrm{LiCl}$ for different polynucleotides with the same length. The raw current traces of $\mathrm{dA}_{20}, \mathrm{dT}_{20}$ and $\mathrm{dC}_{20}$ show more frequent blockades acquired in $\mathrm{LiCl}$ than $\mathrm{KCl}$ (Fig. 4a). The event frequencies are $8.75 \pm 0.06 \mathrm{~s}^{-1}$ for $\mathrm{dA}_{20}, 9.19$ 
a
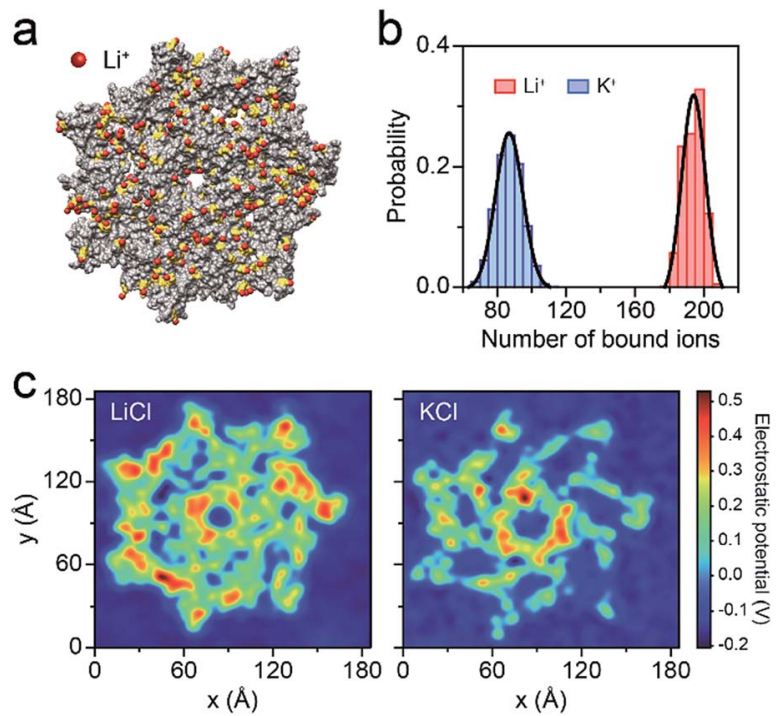

Fig. 3 Molecular dynamics (MD) simulations of AeL in $\mathrm{M}^{+} \mathrm{Cl}^{-}\left(\mathrm{M}^{+}=\mathrm{Li}^{+}\right.$ or $\mathrm{K}^{+}$). (a) $\mathrm{Li}^{+}$bound to the AeL in MD simulations. $\mathrm{Li}^{+}$are represented in red, the negatively-charged amino acids are shown in yellow and the AeL is represented in gray. The illustration is not to scale. (b) The number of $\mathrm{M}^{+}\left(\mathrm{M}^{+}=\mathrm{Li}^{+}, \mathrm{K}^{+}\right)$bound to the AeL. The ions are regarded as being bound within $3.1 \AA$ from the heavy atoms of the AeL. The binding numbers of $\mathrm{Li}^{+} / \mathrm{K}^{+}$are fitted into a Gaussian function. (c) Average electrostatic potential around the cis entrance of the AeL.
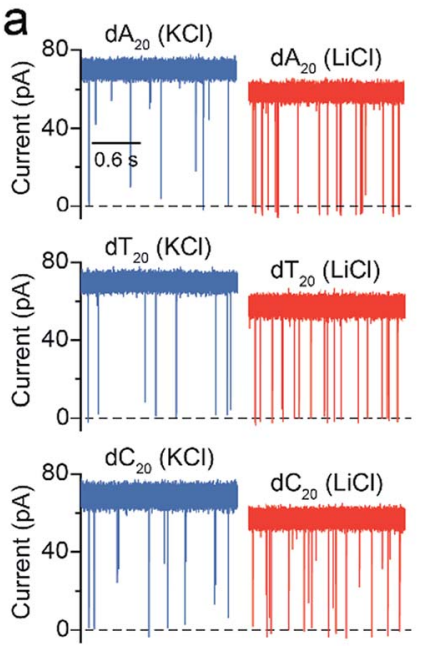
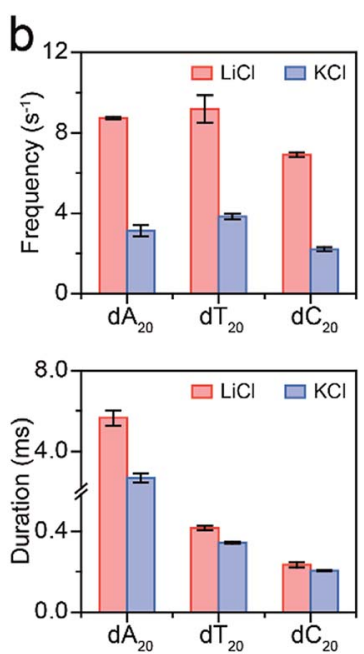

Fig. 4 Analysis of different polynucleotides with the same length in $\mathrm{M}^{+} \mathrm{Cl}^{-}\left(\mathrm{M}^{+}=\mathrm{Li}^{+}\right.$or $\left.\mathrm{K}^{+}\right)$. (a) Raw current traces of $\mathrm{dA}_{20}, \mathrm{dT}_{20}$ and $\mathrm{dC}_{20}$. (b) The event frequencies and durations of $\mathrm{dA}_{20}, \mathrm{dT}_{20}$ and $\mathrm{dC}_{20}$. Event frequencies reveal obvious enhancements for these ssDNA in $\mathrm{LiCl}$. The values of event frequencies and durations were extracted from transformed single-exponential functions (Fig. S7 $\dagger$ ). Data were obtained at $+160 \mathrm{mV}$ in $1 \mathrm{M} \mathrm{M}^{+} \mathrm{Cl}^{-}\left(\mathrm{M}^{+}=\mathrm{Li}^{+}\right.$or $\left.\mathrm{K}^{+}\right), 1 \mathrm{mM}$ EDTA, $10 \mathrm{mM}$ Tris buffer, and $\mathrm{pH} 8.0$ with the presence of $2.0 \mu \mathrm{M}$ ssDNA at the cis side of the AeL nanopore.

$\pm 0.68 \mathrm{~s}^{-1}$ for $\mathrm{dT}_{20}$, and $6.91 \pm 0.11 \mathrm{~s}^{-1}$ for $\mathrm{dC}_{20}$ at $160 \mathrm{mV}$ in LiCl, providing a 2.39-to-3.15-fold increase compared to those in $\mathrm{KCl}$, respectively (Fig. 4a). The difference in event frequencies might result from the structural variations of $\mathrm{dA}_{20}, \mathrm{dT}_{20}$ and $\mathrm{dC}_{20}$ induced by the cation-ssDNA interactions. The duration times are $5.65 \pm 0.38 \mathrm{~ms}$ for $\mathrm{dA}_{20}, 0.42 \pm 0.01 \mathrm{~ms}$ for $\mathrm{dT}_{20}$, and $0.23 \pm 0.01 \mathrm{~ms}$ for $\mathrm{dC}_{20}$ at $160 \mathrm{mV}$ in LiCl (Fig. $4 \mathrm{~b}$ ). The duration differences in LiCl between $\mathrm{dA}_{20}$ and $\mathrm{dT}_{20}, \mathrm{dA}_{20}$ and $\mathrm{dC}_{20}$, and $\mathrm{dT}_{20}$ and $\mathrm{dC}_{20}$ are $5.23 \pm 0.39,5.42 \pm 0.39$ and $0.19 \pm 0.02 \mathrm{~ms}$, respectively. In $\mathrm{KCl}$, the differences between $\mathrm{dA}_{20}$ and $\mathrm{dT}_{20}, \mathrm{dA}_{20}$ and $\mathrm{dC}_{20}$, and $\mathrm{dT}_{20}$ and $\mathrm{dC}_{20}$ are $2.34 \pm 0.24,2.48 \pm 0.24$ and $0.14 \pm 0.02 \mathrm{~ms}$, respectively (Fig. $4 \mathrm{~b}$ ). Obviously, these values in $\mathrm{KCl}$ are smaller than those gained in $\mathrm{LiCl}$, implying that $\mathrm{LiCl}$ provides a higher discrimination ability in the duration time for AeL with polynucleotides. Moreover, we found that $\mathrm{LiCl}$ leads to a 1.65-fold increase in event frequency for $\mathrm{dA}_{40}$ at $160 \mathrm{mV}$ using an $\alpha$-HL (Fig. S8 $\dagger$ ), which is smaller than the 2.69-fold increase obtained by the AeL. It is reasonable as more negative charges were screened at the cis entrance of the AeL due to the different charge distributions of the two pores. ${ }^{11,25}$

\section{Conclusion}

In conclusion, we have presented a novel and robust method based on LiCl to enhance AeL capture of long ssDNA. By introducing LiCl, the AeL achieved the detection of a ssDNA longer than $100 \mathrm{nt}$ for the first time. LiCl increased the ssDNA capture ability by 2.63 to 10.23 folds (i.e. $60 \mathrm{nt}$ ) compared with $\mathrm{KCl}$ at the same potential and DNA concentration. The calculations demonstrated that $\mathrm{LiCl}$ leads to an experimental reduction in activation energy of $4.48 k_{\mathrm{B}} T$ for $\mathrm{dA}_{60}$ in LiCl. Further MD simulations revealed that the strong $\mathrm{Li}^{+}-\mathrm{AeL}$ binding induces a great screen of negative charges on the pore surface, resulting in electrostatic focusing of long ssDNA into the AeL. Our previous work has shown that the event frequency of a $30 \mathrm{nt}$ ssDNA is relatively low $\left(\sim 0.6 \mathrm{~s}^{-1}\right)$ in $1 \mathrm{M} \mathrm{MgCl}_{2}$, and the effect of divalent cations might be less favorable than monovalent cations for DNA capture. This general strategy is simple and practical without exposing the analyte and nanopore to extreme conditions (i.e. high temperatures and low $\mathrm{pH}$ ). This work would facilitate various real applications of AeL in life science, such as genetic and epigenetic detection, enzyme activity evaluation and signaling pathway description. These findings also provide new insight into the construction of highly-sensitive AeL arrays for drug separation and screening, and direct DNA sensing and sequencing at high throughput and without labelling.

\section{Conflicts of interest}

There are no conflicts to declare.

\section{Acknowledgements}

This research was supported by the "Chen Guang" Project from the Shanghai Municipal Education Commission and Shanghai Education Development Foundation (17CG27), National Natural Science Foundation of China (21421004 and 61871183) and the Fundamental Research Funds for the Central Universities (222201718001, 222201717003, 222201714012). 


\section{Notes and references}

1 R. Stefureac, Y. T. Long, H. B. Kraatz, P. Howard and J. S. Lee, Biochemistry, 2006, 45, 9172-9179.

2 F. Piguet, H. Ouldali, M. Pastoriza-gallego, P. Manivet, J. Pelta and A. Oukhaled, Nat. Commun., 2018, 9, 996.

3 G. Baaken, I. Halimeh, L. Bacri, J. Pelta, A. Oukhaled and J. C. Behrends, ACS Nano, 2015, 9, 6443-6449.

4 C. Merstorf, B. Cressiot, M. Pastoriza-gallego, A. Oukhaled, J.-M. Betton, L. Auvray and J. Pelta, ACS Chem. Biol., 2012, 7, 652-658.

5 B. Zhou, Y. Wang, C. Cao, D. Li and Y. Long, Sci. China: Chem., 2018, 61, 1-4.

6 Y. Wang, L. Q. Gu and K. Tian, Nanoscale, 2018, 10, 1385713866.

7 C. Cao, Y. L. Ying, Z. L. Hu, D. F. Liao, H. Tian and Y. T. Long, Nat. Nanotechnol., 2016, 11, 713-718.

8 C. Cao, J. Yu, M. Li, Y. Wang, H. Tian and Y. Long, Small, 2017, 13, 1702011.

9 Y. Ying, Z. Li, Z. Hu, J. Zhang, F. Meng, C. Cao, Y. T. Long and H. Tian, Chem, 2018, 4, 1893-1901.

10 J. Clarke, H. Wu, L. Jayasinghe, A. Patel, S. Reid and H. Bayley, Nat. Nanotechnol., 2009, 4, 265-270.

11 L. Song, M. R. Hobaugh, C. Shustak, S. Cheley, H. Bayley and J. E. Gouaux, Science, 1996, 274, 1859-1866.

12 M. Faller, M. Niederweis and G. E. Schulz, Science, 2004, 303, 1189-1193.

13 E. A. Manrao, I. M. Derrington, A. H. Laszlo, K. W. Langford, M. K. Hopper, N. Gillgren, M. Pavlenok, M. Niederweis and J. H. Gundlach, Nat. Biotechnol., 2012, 30, 349-353.

14 J. Yu, C. Cao and Y. Long, Anal. Chem., 2017, 89, 1168511689.

15 L. Payet, M. Martinho, C. Merstorf, M. Pastoriza-gallego, J. Pelta, V. Viasnoff, L. Auvray, M. Muthukumar and J. Mathé, Biophys. J., 2015, 109, 1600-1607.

16 C. Cao, M. Li, N. Cirauqui, Y. Wang, M. D. Peraro, H. Tian and Y. Long, Nat. Commun., 2018, 9, 2823.

17 M. Pastoriza-Gallego, M.-F. Breton, F. Discala, L. Auvray, J.-M. Betton and J. Pelta, ACS Nano, 2014, 8, 11350-11360.
18 D. Liao, C. Cao, Y. Ying and Y. T. Long, Small, 2018, 14, 1704520.

19 Y. Wang, K. Tian, X. Du, R. Shi and L. Gu, Anal. Chem., 2017, 89, 13039-13043.

20 N. A. W. Bell, K. Chen, S. Ghosal, M. Ricci and U. F. Keyser, Nat. Commun., 2017, 8, 380.

21 N. A. W. Bell, M. Muthukumar and U. F. Keyser, Phys. Rev. E, 2016, 93, 022401.

22 Y. Zhang, G. Wu, W. Si, J. Ma, Z. Yuan, X. Xie, L. Liu, J. Sha, D. Li and Y. Chen, Nanoscale, 2017, 9, 930-939.

23 S. W. Kowalczyk, D. B. Wells, A. Aksimentiev and C. Dekker, Nano Lett., 2012, 12, 1038-1044.

24 D. Xi, Z. Li, L. Liu, S. Ai and S. Zhang, Anal. Chem., 2018, 90, 1029-1034.

25 I. Iacovache, S. De Carlo, N. Cirauqui, M. Dal Peraro, F. G. van der Goot and B. Zuber, Nat. Commun., 2016, 7, 12062.

26 G. Maglia, M. R. Restrepo, E. Mikhailova and H. Bayley, Proc. Natl. Acad. Sci. U. S. A., 2008, 105, 19720-19725.

27 Y. Wang, C. Cao, Y. Ying, S. Li, M. Wang, J. Huang and Y. Long, ACS Sens., 2018, 3, 779-783.

28 Y. Wang, M. Li, H. Qiu, C. Cao, M. Wang, X. Wu, J. Huang, Y. Ying and Y. T. Long, Anal. Chem., 2018, 90, 7790-7794.

29 M. Boukhet, F. Piguet, H. Ouldali, M. Pastoriza-gallego, J. Pelta and A. Oukhaled, Nanoscale, 2016, 8, 18352-18359.

30 S. Bhattacharya, J. Muzard, L. Payet, J. Mathé, U. Bockelmann, A. Aksimentiev and V. Viasnoff, J. Phys. Chem. C, 2011, 115, 4255-4264.

31 M. Wanunu, W. Morrison, Y. Rabin, A. Y. Grosberg and A. Meller, Nat. Nanotechnol., 2010, 5, 160-165.

32 Y. Wang, K. Tian, L. L. Hunter, B. Ritzo and L. Gu, Nanoscale, 2014, 6, 11372-11379.

33 S. E. Henrickson, M. Misakian, B. Robertson and J. J. Kasianowicz, Phys. Rev. Lett., 2000, 85, 3057-3060.

34 M. Pastoriza-gallego, L. Rabah, G. Gibrat, B. Thiebot, F. G. van der Goot, L. Auvray, J.-M. Betton and J. Pelta, J. Am. Chem. Soc., 2011, 133, 2923-2931.

35 M. Muthukumar, J. Chem. Phys., 2010, 132, 195101. 